\title{
Dynamique de l'occupation du sol de la commune de Téssékéré de 1984 à 2015 (Ferlo Nord, Sénégal)
}

\author{
Diarra SYLLA ${ }^{1,2}$, Taibou BA $^{2}$, Mariama Dalanda DIALLO ${ }^{3,4}$, Tamsir MBAYE ${ }^{5}$, Aly \\ DIALLO $^{6}$, Jean Luc PEIRY ${ }^{3,7}$, Aliou GUISSÉ ${ }^{1,3 *}$ \\ ${ }^{1}$ Département de Biologie végétale, Faculté des Sciences et Techniques, Université Cheikh Anta Diop de Dakar, BP. 5005 \\ Fann, Dakar, Sénégal \\ ${ }^{2}$ Centre de Suivi Écologique pour la gestion des ressources naturelles (CSE), Fann Résidence, Rue Léon Gontran Damas, \\ Dakar, Sénégal \\ ${ }^{3}$ Observatoire Hommes-Milieux international (OHMi) Tessékéré, Ferlo, Sénégal \\ ${ }^{4}$ Section Productions Végétales et Agronomie, UFR des Sciences Agronomiques, de l'Aquaculture et des Technologies \\ Alimentaires, Université Gaston Berger, BP. 234, Saint Louis, Sénégal \\ ${ }^{5}$ Centre National de Recherches Forestières, Institut Sénégalais de recherches Agricoles, Bel Air, Dakar, Sénégal \\ ${ }^{6}$ Département d'Agroforesterie, Université Assane Seck de Ziguinchor, BP: 523 Néma, Ziguinchor, Sénégal \\ ${ }^{7}$ CNRS, Unité Mixte Internationale 3189 "Environnement, Santé, Société », Dakar, Sénégal \\ *Auteur correspondant : alguisse@,orange.sn; +221 776389669
}

Mots clés: Occupation du sol, Dynamique, Télédétection, SIG, Téssékéré, Ferlo, Sénégal

Keywords: Land-cover, Dynamics, Remote sensing, SIG, Téssékéré, Ferlo, Sénégal

Publication date 30/06/2019, http://www.m.elewa.org/JAPS

\section{RESUME}

L'objectif de cette étude a été de déterminer l'évolution de l'occupation du sol de la commune de Tessékéré dans le Ferlo situé au Nord du Sénégal entre 1984 et 2015, en utilisant les techniques de télédétection et les Systèmes d'Information Géographiques (SIG). En 2015, 13 modes d'occupation du sol appelés classes ont été identifiées dans la commune : savane boisée, savane arborée, savane arbustive à arborée, savane arbustive, steppe arborée, steppe arbustive à arborée, steppe arbustive, cultures maraîchères, cultures pluvialesjachère, village, mare, zone nue, plantation. Toutes ces classes étaient présentes dans la commune en 1984 à l'exception de la classe plantation. La dynamique spatio-temporelle de la commune a montré que les classes savane arbustive à arborée, savane arbustive et plantation ont connu une évolution progressive tandis que les classes savane arborée, steppe arborée, steppe arbustive et zone nue ont eu une évolution régressive. L'analyse des changements intervenus entre 1984 et 2015 a montré que 27,46\% de la superficie de la commune sont restés à l'état initial ; $65,54 \%$ ont subi des modifications et $7 \%$ se sont converties. Cette étude élargie sur toute la réserve sylvopastorale (aire protégée) permettra d'évaluer l'efficacité des plans de gestion des zones protégées au Ferlo.

The land cover evolution of the Tessekere commune from 1984 to 2015 (North Ferlo, Senegal) 


\begin{abstract}
The objective of this study was to determine the land cover evolution of the Tessékéré commune between 1984 and 2015, by using the techniques of remote sensing and the geographic information system (GIS). In 2015, 13 land cover patterns called classes were identified in the commune: savanna woodland, trees savannas, shrub savanna with trees, shrub savannas, trees steppe, shrub steppe with trees, shrub steppe vegetable crop rainfall crop and fallow, village, pond, bare soil, trees plantation. All classes were present in the commune in 1984 except the trees plantation class. The space-time dynamics analysis showed that three classes of ground occupation have increased their area (shrubby savannas with trees, shrubby savannas and tree plantations) and four have decreased (trees savanna, trees steppe, shrub steppe and bare soil). Study of changes from 1984 to 2015 showed that: $27.5 \%$ of the surface remained unaffected, $65.54 \%$ underwent modifications inside the same ground occupation category, and 7\% experienced a conversion from one category of ground occupation to another one. This study has been widened at the whole "réserve sylvopastorale" protected area which will make it possible to evaluate the effectiveness of the management plans for protected areas in the Ferlo.
\end{abstract}

\section{INTRODUCTION}

Le suivi de la végétation dans les régions aride et semi-aride de l'Afrique tropicale s'est imposé comme une priorité pour les décideurs comme pour les scientifiques depuis la période de sècheresse (1970-1989) généralisée en région sahélienne (Sarr, 2009). Cette longue période de déficit hydrique et la sédentarisation progressive des tribus nomades ont localement causé une dégradation et une surexploitation de ces écosystèmes fragiles et notamment de la savane arbustive (Conedera et al., 2010). La région du Ferlo n'est pas épargnée par ces phénomènes de dégradation et d'anthropisation. En effet, ces derniers sont liés à l'extension des surfaces cultivées, au surpâturage, au déboisement et à l'élagage des arbres en saison sèche, entre autres (Barral, 1982). Cette situation engendre des perturbations parfois profondes des systèmes écologiques sahéliens, parfois fragiles. On parle souvent de dégradation. Cette dégradation se manifeste par des modifications de la structure de la végétation et de la composition floristique du milieu (Akpo et al., 2003) et est préjudiciable aux conditions de vie des populations rurales et à l'économie locale (Diouf et al., 2002). Un des problèmes les plus urgents à résoudre dans le Ferlo et par extension à la zone sahélienne, est la reconstitution des écosystèmes dégradés. Elle doit reposer sur la connaissance de l'état actuel de l'occupation du sol et de sa dynamique. Les études sur la dynamique des écosystèmes qui ont été réalisées au Ferlo sont déjà anciennes (Barral, 1982 ; Grouzis, 1988 ; Hiernaux, 1990 ; Akpo et Grouzis, 1992 ; Poissonet et al., 1992; Akpo et al., 2003). Celles récentes caractérisent la végétation du point de vue statique, l'approche spatiale ayant été très peu utilisée dans ces travaux (Sarr, 2008; Diouf, 2011; Ngom et al., 2013 ; Ndiaye et al., 2014 ; Ndong et al., 2015). Pourtant, cette approche permet non seulement d'évaluer les superficies des différentes formations végétales, mais aussi de suivre leur évolution au cours du temps. De plus, ces études ont été pour la plupart menées dans des aires non protégées du Ferlo. La dynamique au sein des zones protégées a été peu étudiée. Pourtant, la végétation y évolue de façon sensible. Par conséquent, l'étude de ces espaces est essentielle pour l'application des politiques publiques de gestion.L'objectif de ce travail est d'étudier l'état actuel et les changements affectant la végétation sur trois décennies d'une commune totalement intégrée dans la zone sylvopastorale des six forages (aire protégée). Il s'agit de façon plus spécifique: (i) de cartographier l'occupation du sol de la Commune; (ii) d'identifier les différents modes d'occupation des terres de la zone d'étude; (iii) 
d'étudier la dynamique spatio-temporelle de la zone entre 1984 et 2015; pour enfin (iv) analyser les changements intervenus au cours des périodes 1984-2015.

\section{MATERIEL ET METHODES}

3.1 Site d'étude: L'étude a été réalisée dans la commune de Tessékéré (figure 1), situé dans le département de Linguère, région de Louga (Nord du Sénégal) $\left(15^{\circ} 58^{\prime} \mathrm{N}\right.$ et $15^{\circ} 17^{\prime}$ O). Son choix se justifie par sa position géographique au cœur de la zone sahélienne et par la présence de nombreux projets comme celui de la Grande Muraille Verte (GMV), l'installation des unités pastorales, le Programme d'Appui au Développement Agricole et à l'Entreprenariat Rural (PADAER). La superficie communale est de $1852 \mathrm{~km}^{2}$. Le climat est de type sahélien, caractérisé par une pluviométrie comprise entre $300 \mathrm{~mm}$ et 500 mm (série pluviométrique de 1950 à 2013;
ANAMS, 2014). Le relief est caractérisé par une succession de paléo-dunes et de bas-fonds globalement orientées NE-SO. La végétation est composée d'une mosaïque de savanes arborées à arbustives à peuplement plus dense dans les zones de dépressions, et de steppes. La population de Tessékéré compte 10207 habitants (Ndiaye et al., 2014). Les activités économiques sont l'élevage, marqué par son caractère extensif et transhumant, le commerce, l'artisanat et l'agriculture pluviale. La commune de Tessékéré polarise les trois forages hydrauliques de Widou, d'Amaly et de Tessékéré.

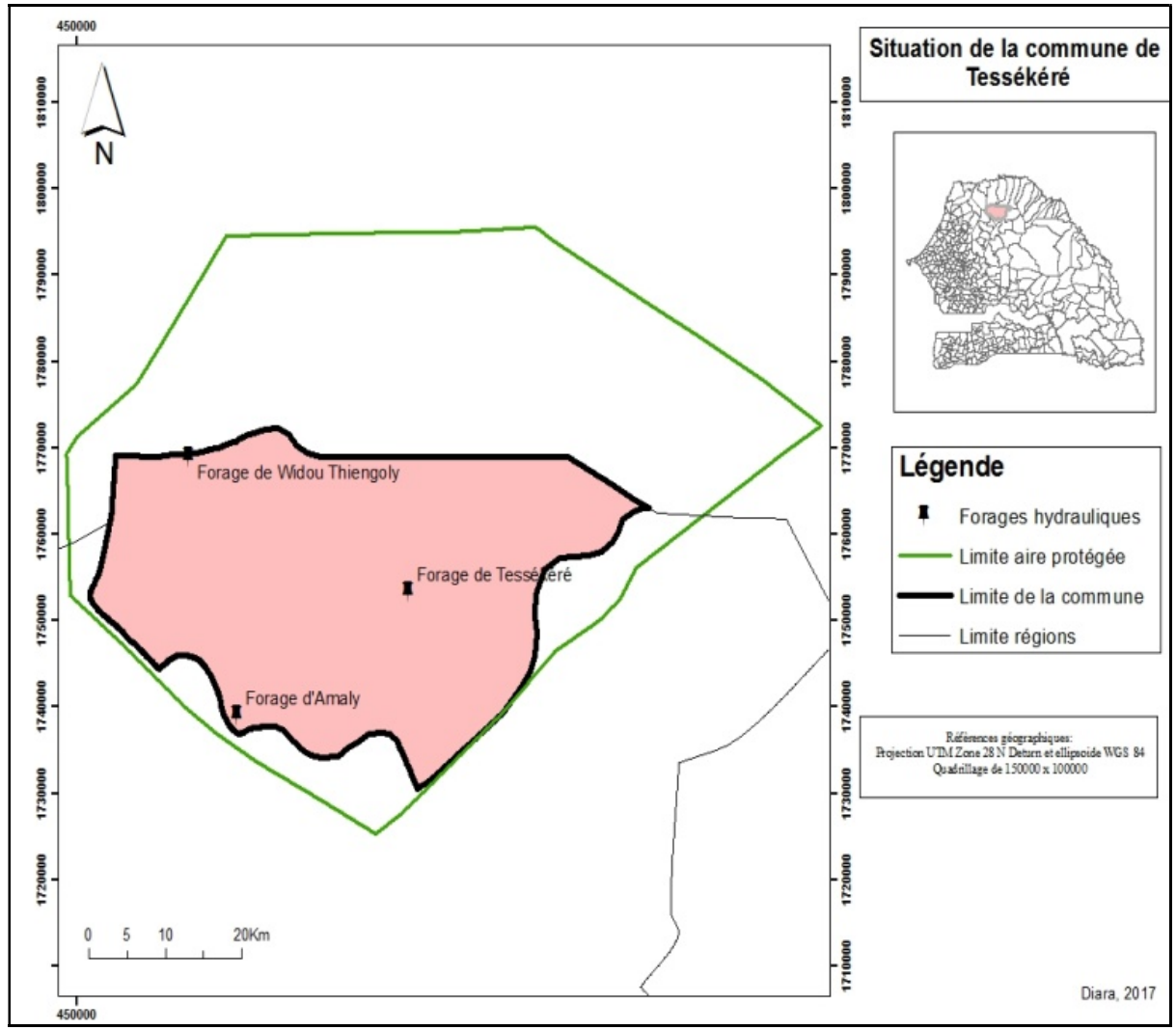

Figure 1: Situation de la commune de Tessékéré

\subsection{Approche méthodologique}




\subsubsection{Données satellitaires et outils} utilisés : Les différentes analyses proviennent de l'exploitation de deux images satellitaires Landsat couvrant toute la zone d'étude. Il s'agit d'images traitées auparavant (corrections radiométriques puis assemblage des scènes) de type TM (Thematics mapper) et ETM+ (Enhanced Thematic Mapper Plus), de résolution $30 \mathrm{~m}$, acquises à une même période de l'année (respectivement le 17 octobre 1984 et le 10 octobre 2013), afin de réduire les problèmes d'interprétation liés aux changements phénologiques de la végétation et aux différences de teinte entre les sols. Selon Stellmes et al. (2010), la résolution $30 \mathrm{~m}$ peut révéler des changements à l'échelle locale. Le traitement de ces images nous permettra donc d'identifier les structures paysagères homogènes propres à notre zone d'étude. Les images sont issues de la base de données du CSE (Centre de Suivi Écologique) de Dakar. Ces images ont été géo référencées dans le système de projection UTM zone $28 \mathrm{~N}$ avec ellipsoïde de référence WGS 84. L'outil est constitué du logiciel SIG ArcGIS pour l'élaboration des cartes d'occupation des sols et de la carte des changements. La classification des formations végétales d'Aubreville (1957) a été utilisée pour l'identification des classes de végétation et leur validation lors des travaux sur le terrain.

\subsubsection{Méthodes de traitements et} d'analyses des images: L'exploitation des images satellitaires a nécessité les activités suivantes: la numérisation, la photointerprétation, les travaux de terrain et la détection des changements intervenus entre 1984 et 2015. Après redressement des images, nous avons procédé à l'interprétation et la numérisation des différents thèmes d'occupation des sols (réseau hydrographique, routiers, localité remarquable...). La numérisation a été faite à l'écran, les objets intéressants ont été digitalisés avec la souris. Le support du zoom permet d'accroître la précision de la délimitation des objets. Comptetenu de la diversité des thèmes à cartographier, de l'objectif final de l'étude et de l'échelle de sortie, nous avons procédé à une photo- interprétation visuelle en nous aidant de la documentation disponible et de la signature spectrale des canaux de l'image. L'interprétation visuelle des images permet l'analyse la plus sûre des différentes structures paysagères qui la composent (Girard, 1973 et 1986), ce qui n'occulte cependant pas des difficultés pour la délimitation précise des différents modes d'occupation du sol (Major et al., 1990).

Les vérifications de terrain ont été effectuées au mois de mai 2015 avec pour objectif de valider la photo-interprétation des différentes formations végétales de l'image de 2013 et d'approfondir l'étude des caractéristiques du milieu. Pour détecter la dynamique du paysage, les vecteurs issus de l'interprétation des deux images ont été superposés pour donner une couche résultante appelée matrice des changements. Cette couche a permis d'identifier les types d'évolution et de calculer les superficies concernées. Ainsi, nous avons distingué :

- les conversions qui correspondent au passage d'une catégorie à une autre, comme par exemple des mares qui se sont transformées en cultures maraîchères ;

- les modifications qui concernent les changements intervenus à l'intérieur d'une même catégorie d'occupation du sol, comme par exemple la dégradation d'une savane arbustive en steppe arbustive ;

- les zones sans changement qui désignent les classes n'ayant été affectées ni par des modifications, ni par des conversions entre les deux dates de l'étude.

Pour faciliter l'analyse, nous avons utilisé des indicateurs environnementaux (Diouf, 2008) pour caractériser l'évolution de l'occupation du sol de la commune. Ces indicateurs sont de deux types (MEPN, 2010) :

- des indicateurs d'équilibre écologique qui regroupent les mares, les savanes arborées et les savanes boisées ;

- des indicateurs de déséquilibre écologique qui regroupent les savanes arbustives, les steppes arbustives à arborées et les steppes arbustives. 
L'organisation de coopération et de développement économiques (OCDE, 1993) définit l'indicateur comme un paramètre (richesse floristique), ou une valeur calculée à partir d'un ensemble de paramètres (capacité de charge) qui fournit des informations sur un phénomène ou sur son état. Le choix de ces indicateurs est pertinent du fait que tous les types d'occupations du sol qui composent ces indicateurs se retrouvent dans notre analyse.

\section{RESULTATS}

4.1 Modes et unités d'occupation des terres : La photo-interprétation des images satellitaires a permis d'identifier 13 classes d'occupation des sols regroupées en cinq catégories (tableau 1): i) les formations végétales naturelles en vert (savane boisée, savane arborée, savane arbustive à arborée, savane arbustive, steppe arborée, steppe arbustive, steppe arbustive à arborée), ii) les zones de cultures en rose clair (cultures maraîchères, cultures pluviales-jachère, plantations), iii) les zones nues en gris, iv) les zones artificialisées en blanc (habitat, village), et v) les surfaces en eau en bleu (mares).

4.2 État de l'occupation du sol de la commune de Tessékéré en 1984 et en 2015 Sur l'image de 1984, 12 classes d'occupation du sol étaient présentes. Elles étaient également sur
Pour chaque scène, les statistiques des différentes unités d'occupation du sol sont présentées en surface relative. Cette dernière est calculée en effectuant le rapport entre la superficie occupée par une classe d'occupation du sol et la superficie de la commune (1853,41 ha). Le bilan d'évolution des différentes unités résulte de la soustraction des deux pourcentages provenant des images 2015 et 1984.

l'image de 2015, auxquelles on a ajouté une nouvelle classe (plantation) au sein de la catégorie «zone de culture». Les statistiques des classes d'occupation du sol de la commune en 1984 et en 2015 sont consignées dans le tableau 1. Les cartes d'occupation du sol (figure 2 et figure 3) présentent la répartition spatiale des différentes unités. En 1984 et en 2015, la catégorie «formation végétale naturelle 》 domine (respectivement 95,3\% et $97 \%$ du territoire en 1984 et en 2015), suivie de la catégorie «zone nue» en 1984 (4,55\%) et de la catégorie "zone de culture» en 2015 (2,66\%). Les catégories «surface en eau» et «zone artificialisée» occupent moins de 1\% en 1984 comme en 2015. 


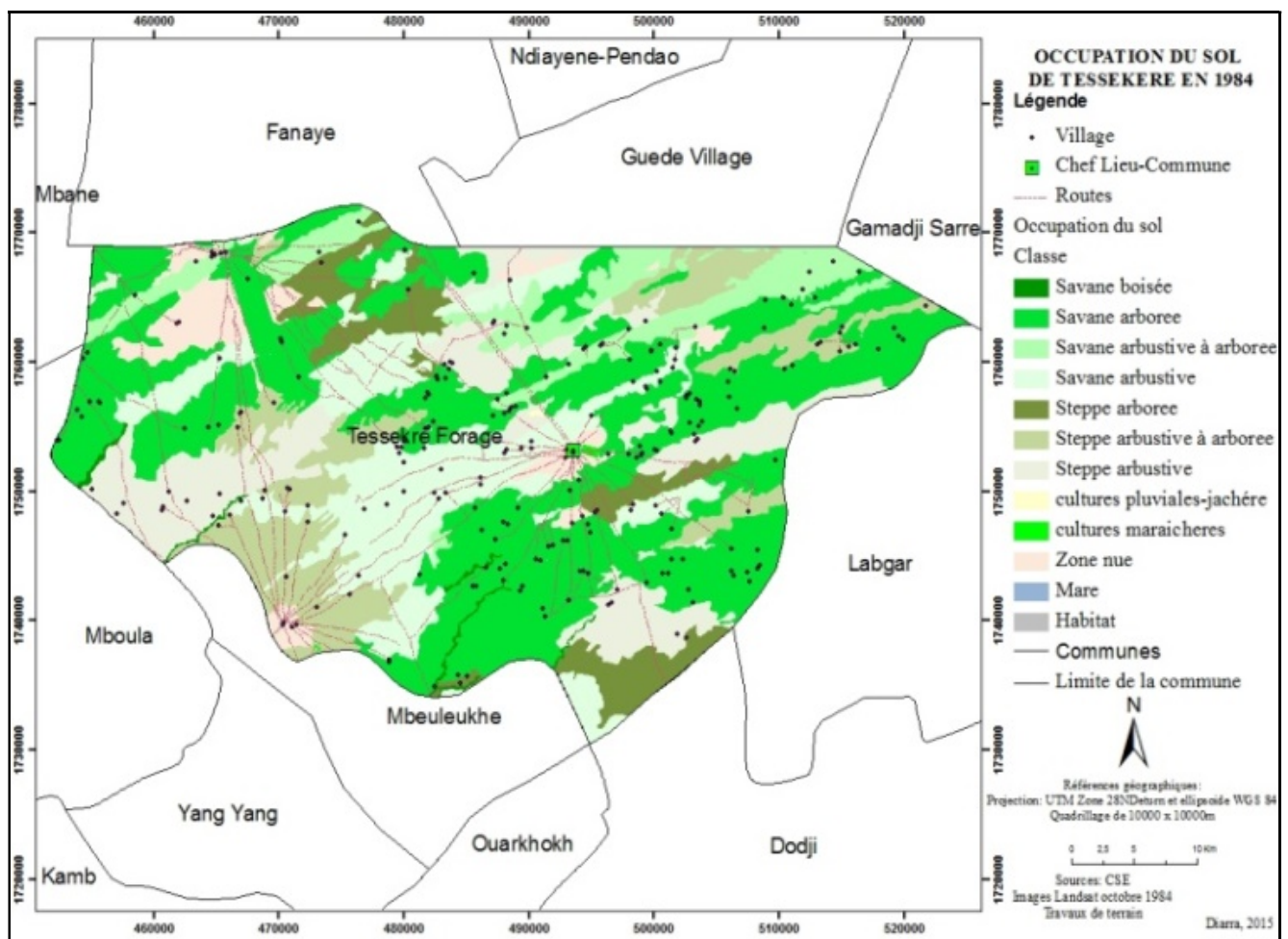

Figure 2 : Carte de l'occupation du sol de la commune de Tessékéré en 1984

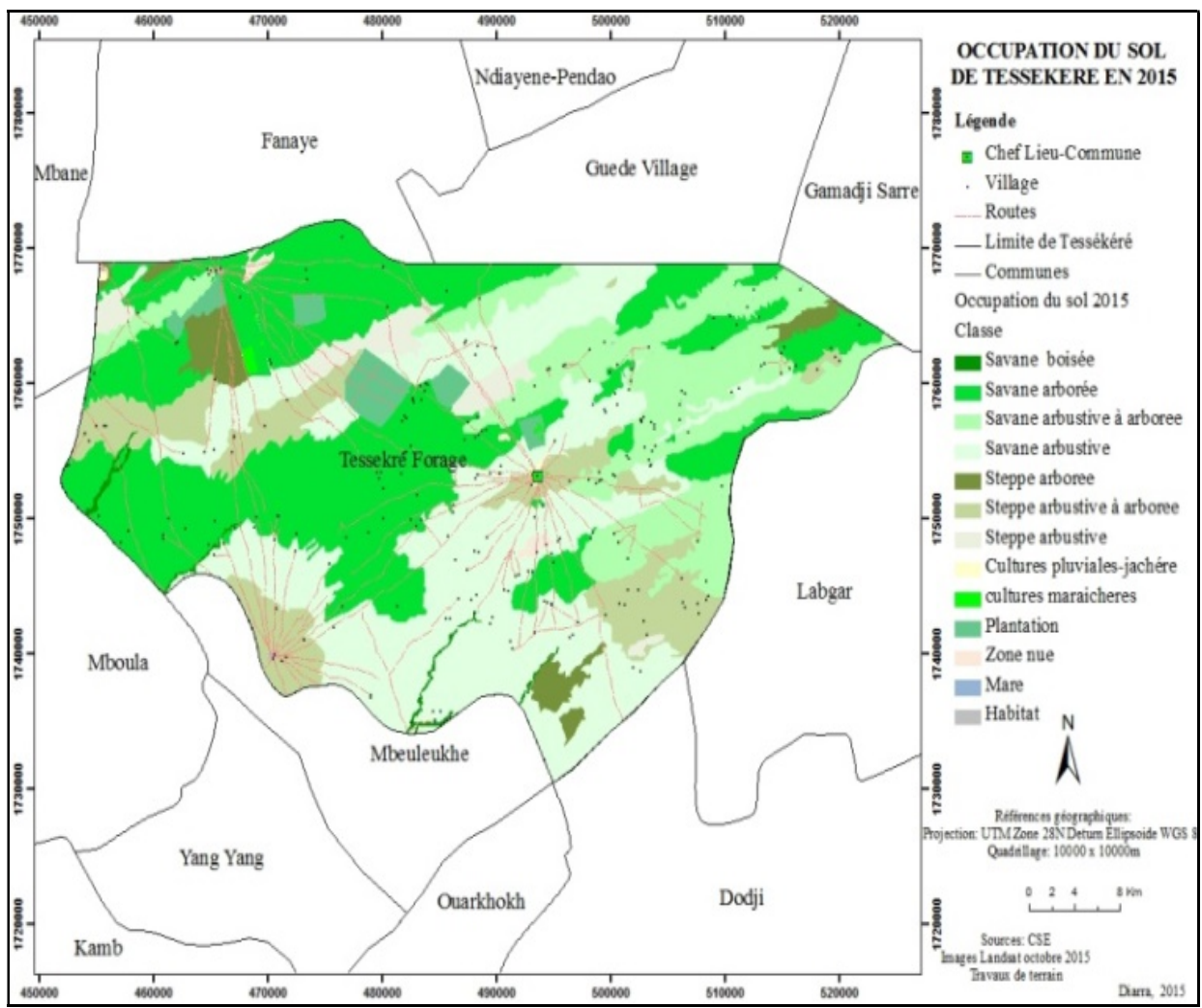

Figure 3 : Carte de l'occupation du sol de la commune de Tessékéré en 2015 
Parmi les types de steppes, la steppe arbustive à arborée domine avec une légère baisse de sa superficie de $0,51 \%$ en 2015 . La steppe arborée est quant à elle passée de $6,11 \%$ à $2,71 \%$ et la steppe arbustive de 13,65\% à 2,78\% entre 1984 et 2015.

Tableau 1 : Statistiques des classes d'occupation du sol en 1984 et en 2015

\begin{tabular}{l|c|c} 
Classes d'occupation des sols & Superficie en $\mathbf{1 9 8 4} \mathbf{( \% )}$ & Superficie en 2015 (\%) \\
\hline Savane boisée & 0,54 & 0,58 \\
Savane arborée & 40,00 & 33,54 \\
Savane arbustive à arborée & 7,89 & 16,67 \\
Savane arbustive & 15,90 & 30,04 \\
Steppe arborée & 6,11 & 2,71 \\
Steppe arbustive à arborée & 11,21 & 10,70 \\
Steppe arbustive & 13,65 & 2,78 \\
Cultures maraîchères & 0,06 & 0,14 \\
Cultures pluviales-jachère & 0,02 & 0,12 \\
Village & 0,05 & 0,08 \\
Mare & 0,002 & 0,02 \\
Zone nue & 4,56 & 0,28 \\
Plantation & - & 2,04 \\
\hline
\end{tabular}

Tableau 2: Statistiques des indicateurs d'état d'équilibre de l'écosystème Tessékéré de 1984 et 2015

\begin{tabular}{l|l|l|l|l|l}
\hline \multicolumn{3}{l}{ Indicateurs d'état d'équilibre écologique } & \multicolumn{3}{l}{ Indicateurs d'état de déséquilibre écologique } \\
\hline $\begin{array}{l}\text { Classes } \\
\text { d'occupation } \\
\text { du sol }\end{array}$ & $\begin{array}{l}\text { Superficie } \\
\mathbf{( 1 9 8 4 )}\end{array}$ & $\begin{array}{l}\text { Superficie } \\
\mathbf{( 2 0 1 5 )}\end{array}$ & $\begin{array}{l}\text { Classes } \\
\text { d'occupation du } \\
\text { sol }\end{array}$ & $\begin{array}{l}\text { Superficie } \\
\mathbf{( 1 9 8 4 )}\end{array}$ & $\begin{array}{l}\text { Superficie } \\
\mathbf{( 2 0 1 5 )}\end{array}$ \\
\hline $\begin{array}{l}\text { Mares } \\
\text { Savane boisée } \\
\text { Savane arborée }\end{array}$ & $\begin{array}{l}0,045 \mathrm{ha} \\
10,08 \mathrm{ha}\end{array}$ & $\begin{array}{l}0,29 \mathrm{ha} \\
10,84 \mathrm{ha} \\
621,69 \mathrm{ha}\end{array}$ & $\begin{array}{l}\text { Savane arbustive } \\
\text { Steppe arbustive } \\
\text { Steppe arbustive à } \\
\text { arborée }\end{array}$ & $\begin{array}{l}294,89 \mathrm{ha} \\
252,90 \mathrm{ha} \\
207,67 \mathrm{ha}\end{array}$ & $\begin{array}{l}556,27 \mathrm{ha} \\
51,63 \mathrm{ha} \\
198,21 \mathrm{ha}\end{array}$ \\
& $750,75 \mathrm{ha}$ & $632,82 \mathrm{ha}$ & Total & $745,46 \mathrm{ha}$ & $806,11 \mathrm{ha}$ \\
\hline
\end{tabular}

La superficie des mares en eau a triplé en 2015 tandis que celle des zones nues a régressé de $4,28 \%$. Dans le même temps, les zones de cultures (cultures pluviales et jachères, cultures maraîchères et plantations) sont passées de $0,08 \%$ à $2,66 \%$ de la superficie communale. Une vue d'ensemble des cartes d'occupation du sol montre trois éléments majeurs (voir figure 2 et 3) : i) le paysage tant en 1984 qu'en 2015 est presque totalement constitué de formations naturelles qui s'organisent en bandes orientées $\mathrm{NE}-\mathrm{SO}$; ii) les savanes arborées ont fortement régressé dans la partie orientale de la commune et ont progressé, mais dans une moindre mesure, dans sa partie Centre-Ouest; iii) le paysage très fragmenté en 1984, s'est fortement simplifié en de grandes unités paysagères en 2015, principalement dans les parties CentreOuest et Nord-Ouest de la commune.

4.3 Bilan de l'évolution des classes d'occupation du sol : L'analyse de l'évolution des classes d'occupation du sol montre que quatre classes d'occupation du sol ont nettement régressé entre 1984 et 2015, de $10,97 \%$ pour la steppe arbustive, de 6,43\% pour la savane arborée, de $3,40 \%$ pour la steppe arborée, et de 4,28\% pour les sols nus. Les savanes arbustives ont, quant à elles, fortement 
progressé $(+14,14 \%)$ et les savanes arbustives à arborées ont gagné 8,78\%. Les plantations d'arbres, qui n'existaient pas en 1984 occupent en $2015,2,40 \%$ de la superficie communale (figure 4). Toutes les autres modes d'occupation du sol ont connu une évolution de surface négligeable.

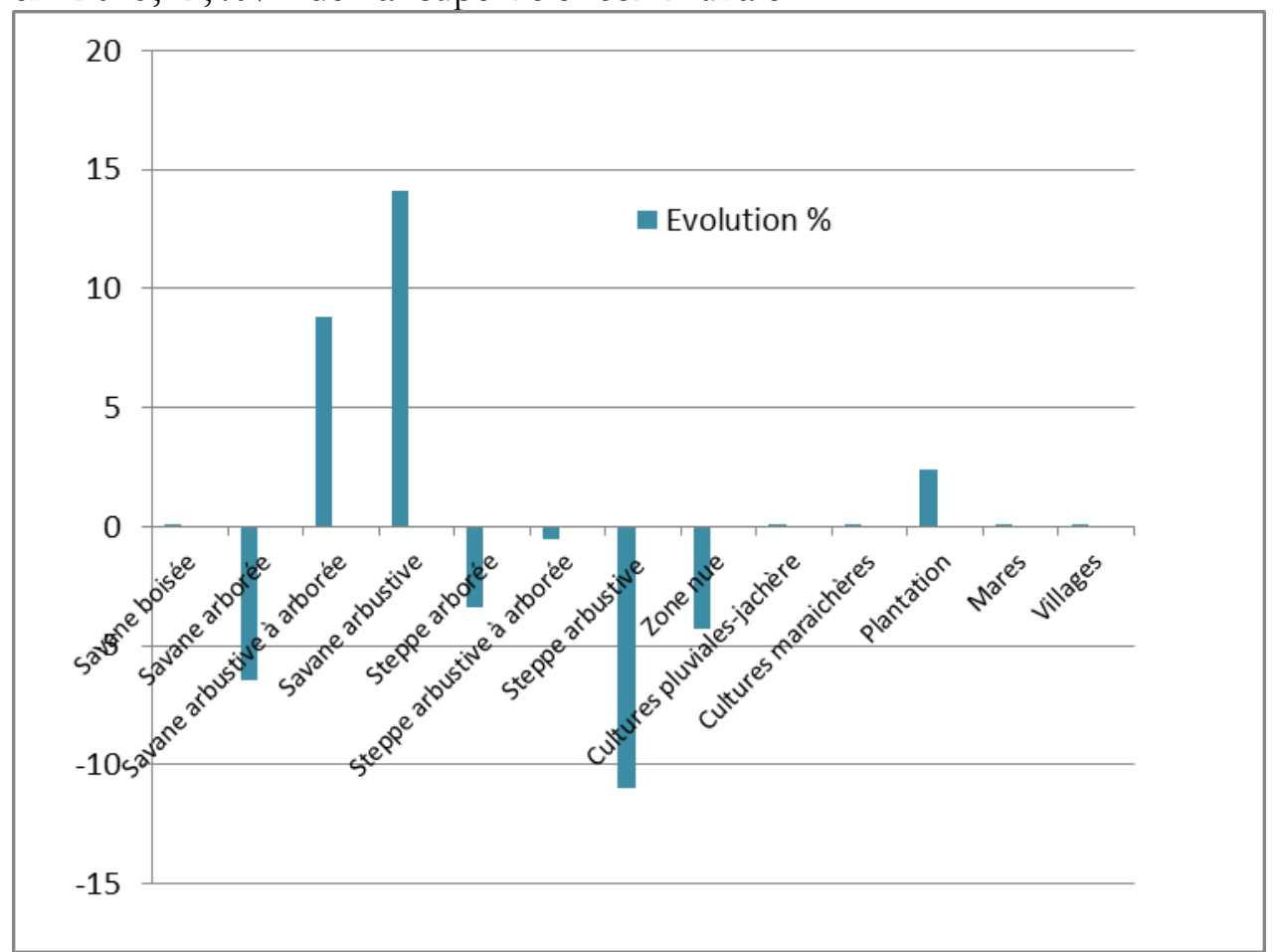

Figure 4: Évolution des superficies des classes d'occupation du sol de la commune de Tessékéré de 1984 à2015

\subsection{Analyse des indicateurs} d'équilibre/déséquilibre de l'occupation du sol : La comparaison des indicateurs d'équilibre (IE) et de déséquilibre écologique (ID) de 1984 et de 2015 montre que l'IE en 1984 est supérieur à celui de 2015 ; de façon inverse, l'ID en 2015 est supérieur à celui de 1984. Nous pouvons conclure de ces deux indicateurs que l'état d'équilibre écologique de la végétation de la commune de Tessékéré s'est dégradé entre 1984 et 2015 et que les classes d'occupations du sol témoignant d'un déséquilibre écologique sont désormais majoritaires.

4.5 Modifications et conversions de l'occupation du sol de la commune de Tessékéré de 1984 à 2015 : Les changements de l'occupation du sol sont synthétisés dans une matrice de transition issue du croisement des cartes d'occupation du sol de 1984 et 2015 (figure 5). Elle identifie les modifications et les conversions. Leur intensité est calculée en divisant la surface d'un type d'occupation du sol en 1984 devenu un autre type en 2015 par la surface totale de celui qu'il est devenu en 2015. Les chiffres représentent donc la fraction de chaque classe qui est passé à une autre, les couleurs permettant d'identifier la dégradation (rouge) de l'amélioration (vert) ; les conversions anthropiques ont également été discriminée bleu clair pour les équilibres et en bleu foncé pour les déséquilibres. Globalement, plus du quart $(27,46 \%)$ du territoire de la commune de Tessékéré n'a connu aucun changement entre les deux dates ; 7,4\% ont connu une conversion $\mathrm{du}$ fait d'une action anthropique directe et 65,5\% concernent les modifications (tableau 3). Les formations végétales naturelles ont ellesaussi évolué, la dégradation l'emportant très nettement sur l'amélioration ( $\mathrm{x} \%$ versus $\mathrm{y} \%$ ) dans les savanes comme dans les steppes. La 
figure 5 représente la matrice de transition qui identifie et mesure l'intensité des changements, des modifications et des conversions. L'intensité de ces changements est calculée en divisant la surface d'un type d'occupation du sol en 1984 devenu un autre type en 2015 par la surface totale de celui qu'il est devenu en 2015.

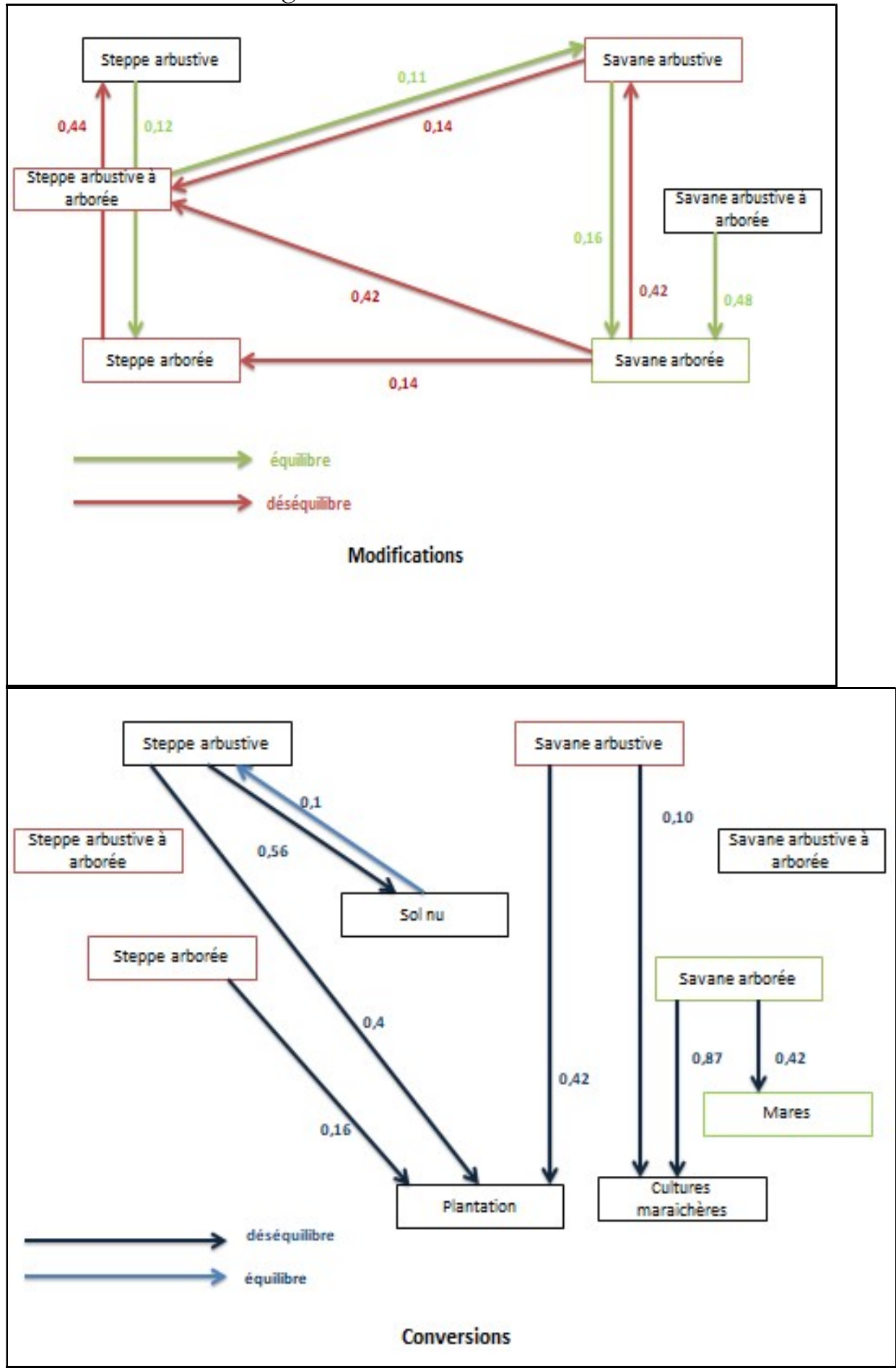

Figure 5: Matrice des changements présentant les modifications et conversions des modes d'occupation du sol de la commune de Tessékéré entre 1984 et 2015 
4.6 Modifications des classes d'occupation du sol de 1984 à 2015 : Les modifications concernent exclusivement les formations végétales naturelles. Ces modifications témoignent des conditions d'équilibre ou de déséquilibre de la végétation. Dans le premier cas, il s'agit de la transformation d'une classe de végétation à une classe plus stable et dans le deuxième cas, c'est une transformation d'une classe de végétation à une classe moins stable. Le tableau 3 montre les différentes transformations subies par la végétation de 1984 à 2015. Les changements qui affectent l'état d'équilibre écologique de la végétation dans la commune de Tessékéré sont majoritaires. Il s'agit de :

- La savane arborée en 1984 s'est dégradée en formations végétales moins stables. En effet, en 2015, 42\% de la steppe arbustive à arborée, $14 \%$ de la steppe arbustive et $42 \%$ de la savane arbustive proviennent de ce qui était une savane arborée en 1984.

- La savane arbustive et la steppe arborée se sont aussi dégradées. En 2015, 44\% de la superficie de la steppe arbustive étaient une steppe arborée en $1984 ; 14 \%$ de la steppe arbustive en 2015 étaient une savane arbustive en 1984. Il a aussi été observé des modifications qui rééquilibrent le processus de dégradation écologique de la végétation. C’est le cas de :

- $\quad 48 \%$ et $16 \%$ de savane arborée en 2015 qui ont été respectivement une savane arbustive à arborée et une savane arbustive en 1984 ;

- $\quad 11 \%$ de savane arbustive en 2015 étaient constituées par une steppe arborée à arbustive ;

- $\quad 12 \%$ de steppe arbustive en 2015 étaient en 1984 une steppe arborée.

4.7 Conversions des classes d'occupation du sol de la commune de Tessékéré de 1984 à 2015 : Les conversions représentent un total de $7 \%$ des changements. Elles sont représentées sur la figure 5. Ces conversions ont affecté l'état d'équilibre mais aussi celui de déséquilibre écologique de la végétation avec une prépondérance de celles affectant l'équilibre du milieu. En effet, en $2015,56 \%$ de la zone nue, $62 \%$ des plantations et $97 \%$ des cultures maraîchères proviennent d'une formation végétale naturelle en 1984. L'unique conversion qui témoigne d'une amélioration du couvert végétal en 2015 est les $10 \%$ de steppe arbustive qui était une zone nue en 1984.

Tableau 3: Bilan des changements de 1984 à 2015

\begin{tabular}{l|c|c|c|c|c|c|c}
\hline \multirow{2}{*}{ Période } & \multicolumn{2}{|c|}{$\begin{array}{c}\text { Superficies sans } \\
\text { changement }\end{array}$} & \multicolumn{2}{|c|}{ Superficies modifiées } & \multicolumn{2}{|c|}{$\begin{array}{c}\text { Superficies } \\
\text { converties }\end{array}$} & \multirow{2}{*}{ Total (ha) } \\
\cline { 2 - 6 } & (ha) & $\%$ & (ha) & $\%$ & (ha) & $\%$ & \\
\hline $1984-2015$ & 50805,62 & 27,46 & 121380,43 & 65,54 & 12511,26 & 7 & 184989,003 \\
\hline
\end{tabular}

\section{DISCUSSION}

La comparaison des indicateurs d'équilibre (IE) et des indicateurs de déséquilibre (ID) de Tessékéré en 1984 et en 2015 montre que le couvert végétal de la commune de Tessékéré, malgré une progression relative de la végétation dans le Ferlo (Sarr, 2009) a subi une dégradation entre ces deux dates. Ces résultats confirment ceux de $\mathrm{Ba}$ (2011), et du CSE (2014) qui indiquaient respectivement l'état de dégradation du couvert végétal de la commune de Barkedji de 1973 à 2011 et de «'unité pastorale de Tessékéré de 2004 à 2014. Ces résultats peuvent être expliqués par de multiples facteurs. Depuis plusieurs décennies, ces milieux subissent de fortes perturbations liées d'une part à l'aridité caractérisée par une longue saison sèche, une forte évaporation, de faibles précipitations et de fortes variabilités spatiotemporelles (Le Houerou, 1989; Ba, 2011 ; CSE, 2014) et d'autre part par une démographie galopante qui a accru la pression (CSE, 2014). De plus, la réduction des aires pâturables, l'augmentation du bétail et la saturation de l'espace ont considérablement accru la pression sur les ressources fourragères accentuant ainsi leur dégradation et la diminution (quantité et 
qualité) de la biomasse disponible pour les troupeaux (Yung et Bosc, 1992 ; Sarr, 2009; CSE, 2014). Le surpâturage se développe, tandis que les pressions sur les ressources ligneuses sont de plus en plus importantes (Bellefontaine et al., 1997 ; Ndong, et al., 2015). En zone sahélienne, ces modifications touchent tout particulièrement les parcours pastoraux (Hiernaux et al., 1990; Diouf, 2011; Ndong et al., 2015). Les résultats de notre étude ont montré que les conversions avec amélioration de la situation écologique ont surtout affecté les zones nues (près de 4000 ha). Le recul des sols nus peut s'expliquer d'une part par la mise en place du Projet Sénégalo-Allemand GTZ intitulé : Modèle d'Aménagement Sylvopastoral des terres de la Zone Nord à partir de 1986 et qui a affecté l'extrémité NO de la commune (voir figure 6), et d'autre part par un reverdissement suite à l'augmentation de la pluviométrie (Sarr, 2009). Ces résultats corroborent ceux de (Bakhoum, 2013) qui indique, que les parcours communautaires de Téssékéré, soumis depuis la sécheresse de 1970 à une instabilité pluviométrique mais aussi aux pressions anthropiques, arrivent de nos jours à se restaurer avec une amélioration de la pluviométrie. De même, Ruster (2009) a établi que la progression du couvert végétal, subie par la zone de Téssékéré de 1988 à 2000, coïncide avec une augmentation de la pluviométrie au Ferlo et la dégradation de 2000 à 2010 à une baisse de la pluviométrie. Pour les zones de cultures qui sont passées de $0,08 \%$ à $2,66 \%$, la progression est largement due aux plantations de la Grande Muraille Verte (initiative panafricaine pour la reforestation du Sahel qui a vu le jour en 2007). Les cultures pluvialesjachère et les cultures maraîchères sont quasi inexistantes dans cette commune de la Réserve Sylvopastorale des Six Forages, totalement vouée à l'élevage. Nos résultats montrent une augmentation du nombre de mares par rapport à 1984. Ils confirment ceux de Sy (2009) et de l'observatoire du Sahel et du Sahara (2013) pour qui cet accroissement serait dû à l'aménagement et la création de nouvelles mares pour répondre à la forte demande hydrique de la zone suite au désengagement de l'état dans la gestion des forages. Ces auteurs rapportent également la perception des pasteurs d'une augmentation de la disponibilité en mares, en liaison plus étroite avec la présence de vallées fossiles et de condition pluviométriques meilleures. 


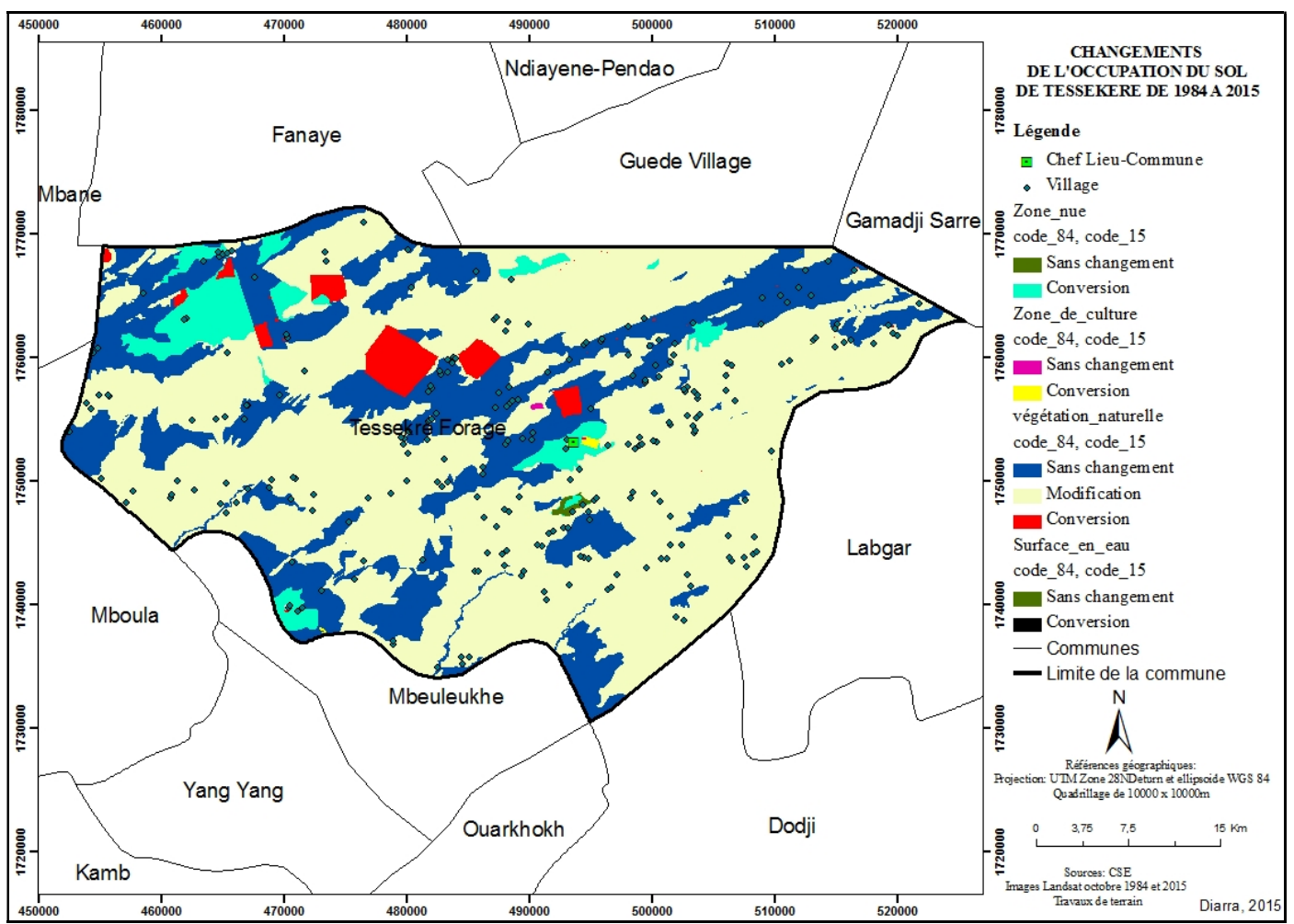

Figure 6: Carte des changements de l'occupation du sol de la commune de 1984 et de 2015

Pour ce qui concerne les formations naturelles, les patches de savane boisée ont été l'unité la moins perturbée entre les deux dates. Elles se situent au niveau des dépressions où les conditions de sécheresse sont les moins critiques. La diminution de la savane arborée au profit des savanes arbustive et arbustive à arborée (signe de dégradation) peut s'expliquer par l'irrégularité de la pluviométrie, même si elle a globalement augmenté par rapport aux décennies de sécheresse. L'une des manifestations de la dégradation est la réduction de la densité de la végétation (Piot et Diaité, 1983) et l'apparition d'espèces plus adaptées aux irrégularités pluviométriques (OSS, 2013), imputables respectivement aux conditions climatiques et aux actions anthropiques. Ces résultats corroborent ceux de Touré (2010), qui avait utilisé l'approche perception paysanne, montrant que les populations avaient constaté une dégradation $\mathrm{du}$ couvert végétal et une diminution du pâturage après divers évènements (sécheresse, feu de brousse, surpâturage). Pour Hountondji et al. (2009), ce phénomène s'explique par l'augmentation de la population et les impacts qu'ils induisent sur les ressources naturelles. Le rapprochement des variables démographiques et économiques met aussi en évidence un déséquilibre entre les besoins des populations et les ressources disponibles (Onibon, 1999). Il en résulte un amenuisement de la capacité productive des terres d'année en année et une augmentation de la précarité et de la pauvreté des populations rurales. Les feux représentent ainsi une cause de destruction des milieux forestiers (Aubreville, 1953; Kouadio, 2013), mais la majorité des scientifiques admettent aussi qu'ils peuvent constituer un facteur de maintien pour les savanes (Aubreville, 1953; Sonko, 2000). Cette idée correspond à la notion «d'incorporation» par l'écosystème végétal d'une perturbation récurrente (Allen et Starr, 1982 ; Fournier, 2001). Un certain contrôle rendrait possible leur régulation et leur stabilisation. La suppression totale des feux ou la pratique des feux précoces ferait évoluer les savanes vers des milieux plus fermés et 
modifierait la physionomie et la composition de la végétation, ainsi que l'équilibre de l'écosystème dans son ensemble. Ainsi, l'accroissement substantiel de la superficie des savanes arbustives que nos données ont révélé est peut être aussi révélateur d'un meilleur contrôle des feux. L'augmentation de la steppe peut être expliquée par la colonisation d'espèces plus tolérantes aux irrégularités pluviométriques, par exemple l'apparition de Calotropis procera dans la zone (OSS, 2013). Elles reposent sur des phénomènes de résistance au déficit hydrique et de compétition qui se déroulent au cours de son établissement (Grouzis, 1988), mais également sur les modifications des écosystèmes de savane en steppe, par dégradation. Sur le plan spatial, la recomposition du paysage autour de grands ensembles avec une dégradation des formations végétales dans l'est et une progression des formations arborées à l'Ouest de Tessékéré Forage pourrait être due à la combinaison de facteurs écologiques, climatiques et anthropiques. En effet, il existe un gradient pluviométrique décroissant d'Ouest en Est (Ndiaye et al., 2014 ; Sène et al., 2018), ce qui

\section{CONCLUSION}

L'étude de l'évolution de l'occupation du sol de Tessékéré sur une période de 31 ans a révélé des changements dans les modes d'occupations des terres, qui se sont traduits par une modification très profonde du paysage. La dynamique de l'occupation du sol se manifeste par une régression des indicateurs d'état d'équilibre actuels par rapport à ceux de 1984. L'analyse de l'évolution de l'occupation du sol a montré que $27,5 \%$ de la superficie de la commune sont restés à l'état initial, 65,5\% ont subi des modifications et $7 \%$ des conversions. La zone, identifiée au départ comme une zone dégradée du fait de pressions anthropiques et de sécheresses récurrentes, a continué de se dégrader au cours des 30 dernières années. Les facteurs anthropiques sont prépondérants dans ce processus de dégradation avec des pratiques non conformes à une gestion durable des ressources naturelles. On peut citer entre autres: influe sur la répartition des formations végétales. La remontée du front agricole suite à la saturation du bassin arachidier et la sédentarisation des pasteurs autour des points d'eau, l'histoire du Ferlo boisée (Ndong et al., 2015) pourraient aussi avoir favorisé l'évolution paysagère. Des études plus poussées devront être menées afin de d'approfondir les explications. Au-delà des changements affectant la structure du paysage végétal, les études de Diouf (2011), Bakhoum (2013) et Sène et al. (2018) ont recensé plusieurs espèces disparues et une régénération assurée que par des espèces pionnières (Calotropis procera, Ziziphus mauritiana, Balanites aegyptiaca...) caractéristiques des zones dégradées (Diouf, 2011). De la même façon, Ndiaye et al. (2014) a montré une diversité de la végétation ligneuse et herbacée plus importante au Ferlo Nord qu'au Ferlo Sud. L'existence d'un gradient de richesse du sol Nord-Sud (Ndiaye et al., 2014) et l'appauvrissement des sols suite à une longue période de sécheresse (Bakhoum, 2013) pourraient constituer de solides explications de la diminution de la fragmentation.

l'exploitation à outrance de l'espace pastoral, l'exploitation irrationnelle des ressources forestières, les feux de brousse. L'analyse diachronique à travers la cartographie des changements, est une démarche efficace dans l'évaluation de l'évolution des écosystèmes. Les supports (photographies aériennes, images satellitaires...) anciens et récents, combinés aux outils du Système d'Information Géographique (SIG), sont d'une grande utilité dans cette démarche. L'évaluation correcte de ces changements est fondamentale dans l'élaboration et la mise en place de politiques de protection et de restauration des écosystèmes. A long terme, cette étude permettra de voir l'efficacité des modes de gestion des aires protégées récemment mis en place, comme le Programme d'Urgence pour le Développement Communautaire (PUDC) et l'installation des unités pastorales. Elle pourra fournir des outils 
de réflexion pour les améliorer si les résultats montrent une dégradation des formations

\section{REMERCIEMENTS}

Ce travail a bénéficié d'une aide de l'Etat français gérée par l'Agence Nationale de la Recherche au titre du Labex DRIIHM, programme «Investissements d'avenir » portant

\section{REFERENCES BIBLIOGRAPHIQUES}

Akpo LE et Grouzis M : 1992. Effets de l'arbre sur la production et la qualité des pâturages sahéliens. Rapport : Bilan sur les recherches relatives au sylvopastoralisme au Sahel. UNESCO IRCS - Sahel 1 République d'Allemagne, Dakar.

Akpo LE, Banoin M et Grouzis M : 2003. Effet de l'arbre sur la production et la qualité fourragères de la végétation herbacée : bilan pastoral en milieu sahélien. Revue Méd. Vét. 154 (10) : 619-628.

Allen TF et Starr TH: 1982. Hierarchy: perspectives for ecological complexity. Chicago, The University of Chicago Press. 310 p.

ANAMS : 2014. Agence Nationale de l'Atmosphère et de la Météorologie du Sénégal. Registre d'enregistrement des relevés pluviométriques. $250 \mathrm{p}$.

Aubreville A: 1953. Les expériences de reconstitution de la savane boisée de Cote d'Ivoire. Bois et Forêts des Tropiques $32: 4-10$.

Aubreville A : 1957. Accord à Yangambi sur la nomenclature des types africains de végétation. Bois et Forêts des Tropiques 51 : 23-27.

Ba T: 2011. Dynamique de l'occupation des sols de la communauté rurale de BAKEDJI au Ferlo (Sénégal). Mémoire de DEA, BV, FST, UCAD (Sénégal). 65 p.

Bakhoum A : 2013. Dynamique des ressources fourragères: Indicateurs de résilience des parcours communautaires de Tessékéré au Ferlo, (Nord Sénégal). Thèse unique de doctorat: Biologie, végétales.

la référence ANR-11-LABX-0010. Les auteurs remercient l'OHMi Tessékéré et le Labex DRIIHM pour leur soutien. pathologie et production animale: option: Pastoralisme, UCAD. 118 p.

Barral H : 1982. Le Ferlo des forages: gestion ancienne et actuelle de l'espace, ORSTOM. 85 p.

Bellefontaine R, Gaston A et Petruccy Y : 1997. - Aménagement des forêts naturelles des zones tropicales sèches. Cahier FAO Conservation. 316 p.

Conedera M, Bomio-Pacciorini N, BornioPacciorini P, Sciacca S, Grandi L, Bouleima A et Vettraino AM: 2010. Reconstitution des écosystèmes dégradés sahéliens. Bois et Forêts des tropiques 304 : 61-71.

CSE (Centre de Suivi Écologique): 2014. Centre de suivi écologique. Rapport sur la caractérisation de l'unité pastorale de Tessékéré. 21 p.

Diouf JC: 2011. Dynamique du peuplement ligneux au Ferlo (Nord-Sénégal), Conséquences et perspectives pour une gestion durable. Thèse de Doctorat en biologie végétale: option Ecologie, UCAD. 264 p.

Diouf A : 2008. Interaction Société, Nature et climat au Sahel: La rupture socioéconomique et écologique au CentreEst agrosylvopastoral sénégalais au XXe siècle. Thèse de doctorat en Sciences géographiques, université de Laval, Quebec. 352 p.

Diouf M, Logbo J, Do F. et Akpo LE : 2002. Dynamique du peuplement ligneux d'une végétation sahélienne au NordSenegal (Afrique de l'Ouest). Journal des Sciences 2(1) : 1-8. 
Fournier A : 2001. Végétation des jachères et succession post-culturale en Afrique tropicale. In «La jachère en Afrique tropicale », John Libbey Eurotext, Paris. Pp. 123-168.

Girard MC: 1973. Interprétation des photographies ballon, comparaison avec les petites régions agricoles. Société française Photogrammétrie $52: 23-36$.

Girard CM : 1986. Interprétation pédologique des photos prises par Spacelab. ITC Journal 1 : 14-18.

Grouzis M: 1988. Structure, productivité et dynamique des systèmes écologiques sahéliens: la mare d'Oursi au Burkina Faso. Paris: ORSTOM, Collection Etudes et Thèses. 336 p.

Hiernaux R, Diarra L et Maiga A : 1990. Dynamique de la végétation ligneuse sahélienne après sécheresse. Un bilan du suivi des sites pastoraux au Gourma en 1989. CIPEA-ILCA Mali. Document de travail $\mathrm{n}^{\circ}$ 00/01. $30 \mathrm{p}$.

Le houerou HN: 1989. The grazing land ecosystems of the African Sahel, Springer-Verlag, Berlin. 282 p.

Hountondji YC, Sokpon N, Nicolas J et Ozer P : 2009. Ongoing desertification processes in the Sahelian belt of West Africa: an evidence from the rain-use efficiencies, Recent Advances in Remote Sensing and Geoinformation Processing for Land Degradation Assessment, ISPRS Series (Taylor and Francis). Pp. 173-186.

Kouadio, KB, N'Da DH, Vroh BT, Zobi IC et N'Gessan KE: 2013. Dynamique de végétation et fréquence des feux de brousse dans la réserve de faune d'Abokoumekro (Centre Côte d'Ivoire). European Scientific Journal 9(35) : 179-192

Major DJ, Baret F et Guyot G: 1990. A ratio vegetation index adjusted for soil brightness. International Journal of Remote Sensing. A ratio vegetation index adjusted for soil brightness. International Journal of Remote Sensing 11 : 727-740.
MEPN (Ministére de l'environnement et de la protection de la nature): 2010. Évaluation et tendances des écosystèmes forestiers du Sénégal. Rapport final. 244 p.

Ndiaye O, Diallo A, Wood SA et Guissé A : 2014. Structural Diversity of Woody Species in the Senegalese Semi-Arid Zone-Ferlo. American Journal of Plant Sciences 5: 416-426.

Ngom D, Fall T, Sarr O, Diatta S et Akpo LE: 2013. Caractéristiques écologiques du peuplement ligneux de la réserve de biosphère du Ferlo (Nord Sénégal). J. Appl. Biosci., 65: 5008 - 5023.

Ndong AT, Ndiaye O, Sagna MB, Diallo A, Galop D et Guissé A: 2015. Caractérisation de la végétation ligneuse sahélienne du Sénégal: cas du Ferlo. Int. J. Biol. Chem. Sci. 9(6): 2548-2594.

OCDE : 1993. Corps central d'indicateurs de l'OCDE pour les examens des performances environnementales. Monographie environnementale, $n^{\circ} 83$, Paris.

Onibon P : 1999. Définition d'une politique et d'un programme d'actions pour la gestion de la transhumance " durable ", dans le cadre de la lutte contre la désertification au Bénin. Mission, MEHU, Cotonou, Bénin. 65 p.

OSS (Observatoire du Sahel et du Sahara): 2013. La Surveillance environnementale dans le circum-Sahara: Synthèse régionale écologie (Algérie - Burkina Faso - Kenya - Mali Niger - Sénégal Tunisie) 2012 $\backslash$ OSS. OSS, Tunis. 128 p.

Piot J et Diaite A : 1983. Systéme de production d'élevage au Sénégal. Etude du couvert ligneux. Compte rendu de fin d'étude. C.I.T: Nogent sur Marne, ISRA, Dakar Hann. 30 p.

Poissonet J, Chambris F et Touré I : 1992. Equilibre et déséquilibre des phytocénoses herbacées sahéliennes : influence de la pluviosité annuelle et de la proximité des points d'eau. In : Le Floc'h E. (ed.), Grouzis Michel (ed.), 
Cornet Antoine (ed.), Bille Jean-Claude (ed.). L'aridité : une contrainte au développement : caractérisation, réponses biologiques, stratégies des sociétés. Paris : ORSTOM. Pp 283-296.

Ruster J : 2009. Evolution du couvert végétal de la région de Tessékéré, par le biais de la télédétection. Rapport de stage: Géographie. Paris. Université Diderot, Paris 7.65 p.

Sarr MA : 2008. Variabilité pluviométrique en Afrique de l'Ouest : Dynamique des espaces végétaux à partir des images satellitales. Exemple du bassin versant du Ferlo, article Journées de Climatologie Nantes, 13-14 mars 2008 - Climat et société : Climat et végétation 57-76 p.

Sarr MA : 2009. Evolution récente du climat et de la végétation au Sénégal (cas du Ferlo), Thèse de doctorat, Université Jean Moulin, Lyon3, LCRE UMR 5600 CNRS. 410 p.

Sène AL, Niang K, Faye G, Ayessou N, Sagna $\mathrm{MB}$, Cisse M, Diallo A, Cissé OK, Gueye M et Guissé A : 2014. Identification des usages de Sclerocarya birrea (A. rich) Hoscht Dans la Zone Du Ferlo (Senegal) et évaluation du potentiel biochimique et nutritionnel de son fruit. Afr. J. Food Agric. Nutr. Dev. 18(2): 13470-13489.

Sonko I : 2000. Étude des effets de différents régimes de feux sur la dynamique de la flore et de la végétation ligneuse des plateaux du parc National du Niokolo Koba, Sénégal. 50 p.

Stellmes M, Udelhoven T, Röder A, Sonnenschein R et Hill J: 2010. Dryland observation at local and regional scale - Comparison of Landsat TM/ETM+ and NOAA AVHRR time series. Remote Sensing of Environment 114(10) : 211-225.

Sy O : 2009. Rôle de la mare dans la gestion des systèmes pastoraux sahéliens du Ferlo (Sénégal). Cybergeo: European Journal of Geography $1-23$ p.

Touré I: 2010. Études de cas sur la vulnérabilité Et l'adaptabilité des éleveurs face aux événements dans la commune de Tessékéré au Sénégal. Projet de recherche: Élevage, Climat et Société, ANR. 106 p.

Yung JM et Bosc PM : 1992. Le développement agricole au Sahel. Tome IV: Défis, recherches et innovations au sahel. Collection « Documents systèmes agraires » $\mathrm{N}^{\circ}$ 17, CIRAD. 384 p. 